PAPER Special Section on Discrete Mathematics and Its Applications

\title{
Codeword Set Selection for the Error-Correcting 4b/10b Line Code with Maximum Clique Enumeration
}

\author{
Masayuki TAKEDA $^{\dagger \text { a) }}$, Nonmember and Nobuyuki YAMASAKI ${ }^{\dagger}$, Member
}

\begin{abstract}
SUMMARY This paper addresses the problem of finding, evaluating, and selecting the best set of codewords for the $4 b / 10 b$ line code, a dependable line code with forward error correction (FEC) designed for real-time communication. Based on the results of our scheme [1], we formulate codeword search as an instance of the maximum clique problem, and enumerate all candidate codeword sets via maximum clique enumeration as proposed by Eblen et al. [2]. We then measure each set in terms of resistance to bit errors caused by noise and present a canonical set of codewords for the $4 \mathrm{~b} / 10 \mathrm{~b}$ line code. Additionally, we show that maximum clique enumeration is \#P-hard.

key words: 4b/10b, NP-hard, maximum clique enumeration
\end{abstract}

\section{Introduction}

Most control systems today are designed as distributed realtime systems. Instances of these systems can be found everywhere: in industrial plants, robots, and spacecraft.

In a real-time system, correctness of tasks are defined not only by the correctness of a given task's output but also by the completion of the task before a given deadline. Meeting deadlines is crucial for these systems as failure to do so may result in catastrophic consequences including loss of life. Thus, tasks and the underlying software are subject to strict requirements and schedulability analysis [3] in order to guarantee deadlines.

Modern real-time systems are usually comprised of many real-time systems interoperating in a distributed fashion. Real-time communication, with guarantees on bounds of end-to-end latency is required for building these systems [4]. Retransmission cannot be tolerated in real-time communication as it introduces unbounded latency in transmission.

Choices and tradeoffs made at various abstraction layers found in modern communication protocol stacks are often ill-suited for real-time communication, a notable example being line codes. Indeed, the recent trend among line codes is to improve throughput by adopting longer and longer codeword lengths in order to reduce overhead introduced by encoding. This is exemplified by modern line codes such as $64 \mathrm{~b} / 66 \mathrm{~b}$ and $128 \mathrm{~b} / 132 \mathrm{~b}$ in contrast to older line codes such as $8 b / 10 b$ and NRZI. These line codes are used as the underlying encodings for various serial communication protocols such as 100 Gigabit Ethernet [5] and USB

\footnotetext{
Manuscript received October 22, 2019.

Manuscript revised February 26, 2020.

${ }^{\dagger}$ The authors are with the Department of Information and Computer Science, Faculty of Sciece and Technology, Keio University, Yokohama-shi, 223-8521 Japan.

a) E-mail: mtakeda@ny.ics.keio.ac.jp

DOI: 10.1587/transfun.2019DMP0019
}

3.1 [6]. However, most line codes are problematic to build upon when devising protocols for communication in realtime systems. In general, line codes such as $8 b / 10 b$ are designed with three things in mind: achieve DC-balance, where the resulting number of zeros and ones transmitted are the same over time, clock embedding, i.e. the clock may be extracted from the stream of codewords on the receiving end, and error detection. Additional codewords for transmission control may also be desirable.

Unfortunately, line codes with only these three characteristics implicitly assume either a communication channel with little to no noise or lax latency requirements. Real-time systems often operate in environments where the former does not hold, and it is safe to assume that the latter never is the case. One result of this assumption is that conventional line codes do not incorporate any form of FEC, so must rely on either error correction implemented at higher abstraction layers or retransmission. The problem with adding on error correction is that a single bit error occurring at the line code level is translated to a multiple bit error when decoded, thus requiring block-level error correction codes such as ReedSolomon codes [7]. This necessitates waiting for a whole block of bytes to arrive before performing error correction, introducing latency unacceptable for real-time systems. Retransmission, as noted above, is also not an option as it introduces unbounded latency and, in some cases, makes transmission all but impossible due to exponential decreases in throughput as the bit error rate (BER) increases [8].

The $4 \mathrm{~b} / 10 \mathrm{~b}$ line code, proposed by Suito et al. [9] and standardized in IPSJ-TS 0015:2015 [10] and IEC TR 63094:2017 [11], addresses these issues by adding bit-level error correction at the line code level for single bit error correction and double bit error detection, while preserving DCbalance and facilitating clock recovery. This is achieved by setting several constraints on codewords and searching over the ten bit codeword space in an ad-hoc manner. The 18 codewords found are mapped to four bits and two control characters corresponding to "setup" and "idle".

We have found a set of 20 codewords that satisfy the same constraints on codewords imposed by the protocol [1], and used the two additional codewords to add two more control characters used to implement functional compatibility with $8 \mathrm{~b} / 10 \mathrm{~b}$. Due to the formulation of code search as a Maximum Clique Problem, we know that the set of codes found is the largest possible, but the question remains whether the particular ones found is the best among all code sets of size 20 . 
We answer this by improving upon this work by finding all such sets of 20 codewords and presenting the set of codewords which have the best qualitative characteristics for use as a line code. Finding this set corresponds to solving the Maximum Clique Problem and enumerating all such sets correspond to solving the Maximum Clique Enumeration Problem. Additionally, we show that the problem that we are trying to solve is \#P-hard.

\section{Preliminaries}

When considering MCP and related problems, we are concerned with a simple graph $G=(V, E)$ consisting of a set of vertices $V$ and edges $E=\{\{x, y\} \mid(x, y) \in V \times V\}$. Two vertices $x$ and $y$ are said to be adjacent if $\{x, y\} \in E$. We define $\Gamma(x)$ to be the set of vertices which are adjacent to $x$. A clique in $G$ is a complete subgraph, i.e. a subgraph of $V$ in which all vertices are adjacent to each other. If no vertices can be added to a given clique to make a larger clique, the clique is said to be maximal, and the largest maximal cliques in a graph are referred to as maximum cliques. The problem of finding a maximum clique in a given graph is referred to as the Maximum Clique Problem (MCP) and the problem of enumerating all maximum cliques in a given graph is referred to as the Maximum Clique Enumeration Problem (MCE).

\section{Related Work}

Raahemi proposed an error correction scheme for use with the $64 \mathrm{~b} / 66 \mathrm{~b}$ line code [12] which takes error multiplication properties of $64 \mathrm{~b} / 66 \mathrm{~b}$ into account. Carney and Chandler proposed a line code integrated with a BCH code [13]. A line code integrating Reed-Solomon codes, designed for usage in high-energy physics, has been proposed by Papotti [14]. However, these line codes all result in long decode latencies due to large frames or error correction block sizes and are undesirable for use in real-time communication with strict latency requirements.

MCP is known to be an NP-hard problem and there is extensive literature on approaches to solve this problem [15]. While binary code search formulated as MCP has been studied in the relatively limited scope of Hamming and Johnson graphs [16], [17], we are not aware of prior work applying exact algorithms to the problem motivated by finding codes for designing line codes or related encoding schemes.

We make heavy use of the work by Eblen et al. [2] of applying MCP and MCE algorithms to problems in bioinformatics, the only work we are aware of concerning the enumeration of all maximum cliques.

\section{The $4 \mathrm{~b} / \mathbf{1 0 b}$ Line Code}

We briefly review the $4 \mathrm{~b} / 10 \mathrm{~b}$ line code proposed by Suito et al. [9]. $4 b / 10 b$ is a line code which encodes four bits of data as ten bit codewords and has the following characteristics:

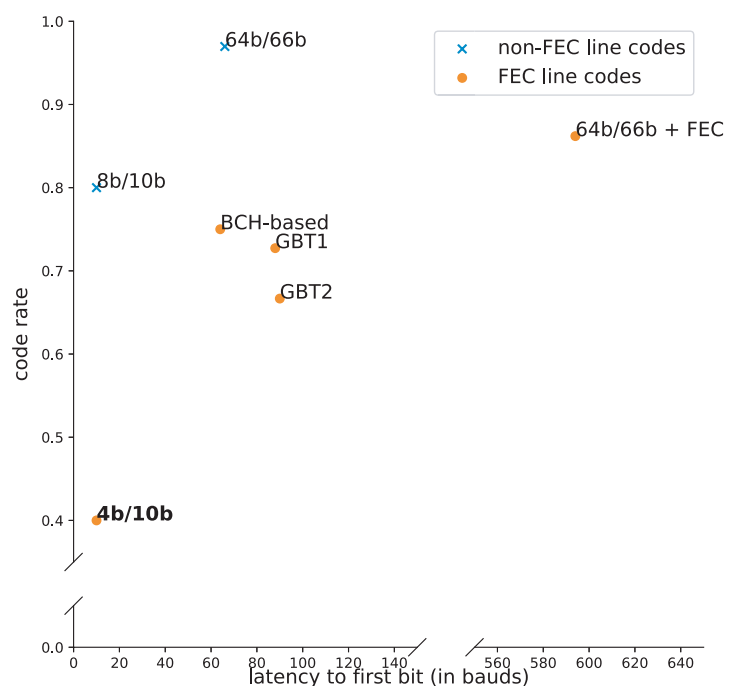

Fig. 1 A comparison of line codes in terms of latency until the first bit is decoded and code rate. In this plot, error-correcting capability is not considered. $4 \mathrm{~b} / 10 \mathrm{~b}$, the line code which is the focus of this work, is highlighted in bold.

Table 1 Machine specifications.

\begin{tabular}{|c|c|}
\hline Processor & Intel Core i7-7600U @ 2.80 GHz \\
\hline Memory & 8x2 GB 2133 MHz DDR4 SODIMM \\
\hline Operating System & Linux 4.19.80, NixOS 19.03 (Koi) \\
\hline Nixpkgs Channel & $19.03 .173677 . d a f 861 \mathrm{a} 810 \mathrm{~d}$ (Koi) \\
\hline Rust Compiler & rustc 1.42.0-nightly (3e0a1c091 2019-12-26) \\
\hline
\end{tabular}

- DC-balance,

- clock recovery,

- single bit error correction and double bit error detection,

- a few control characters for transmission control (two in the case of the original standard, four in the case of our improved version [1]).

This is achieved by selecting codewords which obey the following constraints; for all ten bit codewords $x$,

1. The Hamming weight of $x$ must be five.

2. The run length of $x$ must be less than or equal to five.

3 . The run length of $x$, with a single bit flip in an arbitrary location, must be less than or equal to five.

and for all two codewords $x$ and $y$ where $x \neq y$,

1. The Hamming distance between $x$ and $y$ must be greater or equal to four.

2. The run length of $x y$ must be less than or equal to five.

3 . The run length of $x y$, with two bit flips at least four bits apart, must be less than or equal to five.

where $x y$ is the concatenation of $x$ and $y$, and the run length of $x$ is defined as the length of the longest string of consecutive $0 \mathrm{~s}$ or $1 \mathrm{~s}$ in $x$. For example, the run length of the codeword 1010100011 is three. The error pattern 0000100001 will cause two bit flips four bits apart, changing, for example, the codeword 1010100011 to 1010000010 . This error pattern fulfills the criteria for Constraint 3. An example 
Table 2 Codewords appearing in maximum cliques found (continued in Table 3).

\begin{tabular}{|c|c|}
\hline vertex number & codeword \\
\hline 0 & 0010110101 \\
\hline 1 & 0010110110 \\
\hline 2 & 0010111001 \\
\hline 3 & 0010111010 \\
\hline 4 & 0011001101 \\
\hline 5 & 0011001110 \\
\hline 6 & 0011010011 \\
\hline 7 & 0011100101 \\
\hline 8 & 0011100110 \\
\hline 9 & 0011101001 \\
\hline 10 & 0011101010 \\
\hline 11 & 0100101101 \\
\hline 12 & 0100101110 \\
\hline 13 & 0100110011 \\
\hline 14 & 0100111001 \\
\hline 15 & 0100111010 \\
\hline 16 & 0101001011 \\
\hline 17 & 0101010101 \\
\hline 18 & 0101010110 \\
\hline 19 & 0101011001 \\
\hline 20 & 0101011010 \\
\hline 21 & 0101011100 \\
\hline 22 & 0101100011 \\
\hline 23 & 0101100101 \\
\hline 24 & 0101100110 \\
\hline 25 & 0101101001 \\
\hline 26 & 0101101010 \\
\hline 27 & 0101110001 \\
\hline 28 & 0101110010 \\
\hline 29 & 0101110100 \\
\hline 30 & 0110001011 \\
\hline 31 & 0110001101 \\
\hline 32 & 0110001110 \\
\hline 33 & 0110010101 \\
\hline 34 & 0110010110 \\
\hline 35 & 0110011001 \\
\hline 36 & 0110011010 \\
\hline 37 & 0110011100 \\
\hline 38 & 0110100011 \\
\hline 39 & 0110100101 \\
\hline 40 & 0110100110 \\
\hline 41 & 0110101001 \\
\hline 42 & 0110101010 \\
\hline 43 & 0110110100 \\
\hline 44 & 0111000101 \\
\hline 45 & 0111000110 \\
\hline 46 & 0111001100 \\
\hline 47 & 0111010001 \\
\hline 48 & 0111010010 \\
\hline 49 & 1000101101 \\
\hline
\end{tabular}

of an error pattern that does not is 0000010001 , as the bit flips it will cause are only three bits apart. Constraint 1 enables single bit error correction and double bit error detection. Constraints 2 and 3 enable clock recovery by ensuring a timely bit transition.

We show a comparison between $4 \mathrm{~b} / 10 \mathrm{~b}$ and other line codes in Fig. 1 . Note that while $4 \mathrm{~b} / 10 \mathrm{~b}$ has a significantly lower code rate when compared to other error-correcting line codes, it is the only line code with a decode latency on par with $8 b / 10 b$.
Table 3 Codewords appearing in maximum cliques found (continued from Table 2).

\begin{tabular}{|c|c|}
\hline vertex number & codeword \\
\hline 50 & 1000101110 \\
\hline 51 & 1000110011 \\
\hline 52 & 1000111001 \\
\hline 53 & 1000111010 \\
\hline 54 & 1001001011 \\
\hline 55 & 1001010101 \\
\hline 56 & 1001010110 \\
\hline 57 & 1001011001 \\
\hline 58 & 1001011010 \\
\hline 59 & 1001011100 \\
\hline 60 & 1001100011 \\
\hline 61 & 1001100101 \\
\hline 62 & 1001100110 \\
\hline 63 & 1001101001 \\
\hline 64 & 1001101010 \\
\hline 65 & 1001110001 \\
\hline 66 & 1001110010 \\
\hline 67 & 1001110100 \\
\hline 68 & 1010001011 \\
\hline 69 & 1010001101 \\
\hline 70 & 1010001110 \\
\hline 71 & 1010010101 \\
\hline 72 & 1010010110 \\
\hline 73 & 1010011001 \\
\hline 74 & 1010011010 \\
\hline 75 & 1010011100 \\
\hline 76 & 1010100011 \\
\hline 77 & 1010100101 \\
\hline 78 & 1010100110 \\
\hline 79 & 1010101001 \\
\hline 80 & 1010101010 \\
\hline 81 & 1010110100 \\
\hline 82 & 1011000101 \\
\hline 83 & 1011000110 \\
\hline 84 & 1011001100 \\
\hline 85 & 1011010001 \\
\hline 86 & 1011010010 \\
\hline 87 & 1100010101 \\
\hline 88 & 1100010110 \\
\hline 89 & 1100011001 \\
\hline 90 & 1100011010 \\
\hline 91 & 1100101100 \\
\hline 92 & 1100110001 \\
\hline 93 & 1100110010 \\
\hline 94 & 1101000101 \\
\hline 96 & 1101000110 \\
\hline 97 & 1101001001 \\
\hline & \\
\hline 501001010 \\
\hline 5
\end{tabular}

\section{Codeword Search as a Maximum Clique Problem}

We are interested in finding the largest set of bit strings, or codewords, that obey a set of constraints which give rise to desirable characteristics of the $4 b / 10 b$ line code such as error correction, DC-balance, and clock embedding. Following our scheme [1], we consider the generalized version of this problem by considering arbitrary constraints on individual codewords and arbitrary constraints on pairs of codewords which must hold for all pairs in a candidate set of codewords. This problem can then be formally described in 
Table 4 All maximum cliques found. Refer to Tables 2 and 3 for actual codewords. Vertices 6 and 91, highlighted in bold, form the smallest maximum clique cover. Set 8 is the codeword set specified in [1] and set 7 is the set that we base the proposed canonical $4 \mathrm{~b} / 10 \mathrm{~b}$ translation table upon, both highlighted in bold.

\begin{tabular}{|c|c|}
\hline set number & vertices \\
\hline \multirow{2}{*}{0} & 0569111317202434 \\
\hline & 35425052545661697476 \\
\hline \multirow{2}{*}{1} & 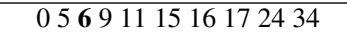 \\
\hline & 35385156576164697478 \\
\hline \multirow{2}{*}{2} & 05569121416182331 \\
\hline & 36384951555862727380 \\
\hline \multirow{2}{*}{3} & 05569131819232631 \\
\hline & 36404953545562727376 \\
\hline \multirow{2}{*}{4} & 14610111516172432 \\
\hline & 35385051565761717479 \\
\hline \multirow{2}{*}{5} & 14610121318192333 \\
\hline & 36414953545562707376 \\
\hline \multirow{2}{*}{6} & $146 \quad 10121416182333$ \\
\hline & 36385155586263707377 \\
\hline \multirow{2}{*}{7} & 14610131720242532 \\
\hline & 35395052545661717476 \\
\hline \multirow{2}{*}{8} & 2567111518192230 \\
\hline & 33405155586263697280 \\
\hline \multirow{2}{*}{9} & 2567131720242531 \\
\hline & 34424953565760687178 \\
\hline \multirow{2}{*}{10} & 3468121417202230 \\
\hline & 34395156576164707179 \\
\hline \multirow{2}{*}{11} & 3468131819232632 \\
\hline & 33415052555860687277 \\
\hline \multirow{2}{*}{12} & 17242535394246485961 \\
\hline & 66747981838588919297 \\
\hline \multirow{2}{*}{13} & 17252834353942465764 \\
\hline & 67757879828690919295 \\
\hline \multirow{2}{*}{14} & 18232636404146475962 \\
\hline & 65738081828687919396 \\
\hline \multirow{2}{*}{15} & 18262733364041465863 \\
\hline & 67757780838589919394 \\
\hline \multirow{2}{*}{16} & 19232833364041465962 \\
\hline & 63738081828688919297 \\
\hline \multirow{2}{*}{17} & 19262937404144485563 \\
\hline & 66727377808490919295 \\
\hline \multirow[b]{2}{*}{18} & 20242734353942465961 \\
\hline & 64747981838587919396 \\
\hline \multirow{2}{*}{19} & 20252937394245475664 \\
\hline & 65717478798489919394 \\
\hline \multirow{2}{*}{20} & 21232636414345475862 \\
\hline & 65727377808487919396 \\
\hline \multirow{2}{*}{21} & 21232836414345475562 \\
\hline & 63737780848688919297 \\
\hline \multirow{2}{*}{22} & 21242535424344485761 \\
\hline & 66717478798488919297 \\
\hline \multirow{2}{*}{23} & 21242735424344485661 \\
\hline & 64747879848587919396 \\
\hline
\end{tabular}

the following way: given a unary relation $A \subseteq V$ and a binary symmetric relation $B \subseteq V \times V$ where the set $V=\{0,1\}^{n}$ is the set of all bit strings of length $n$,

$$
\begin{array}{ll}
\underset{S}{\operatorname{maximize}} & |S| \\
\text { subject to } & S \subseteq A, S \times S \subseteq B .
\end{array}
$$

One solution to this problem can be found by solving MCP on a corresponding graph. By setting $V=A$ and $E=$ $\{\{x, y\} \mid(x, y) \in B\}$, a maximum clique $S$ in $G$ will yield a

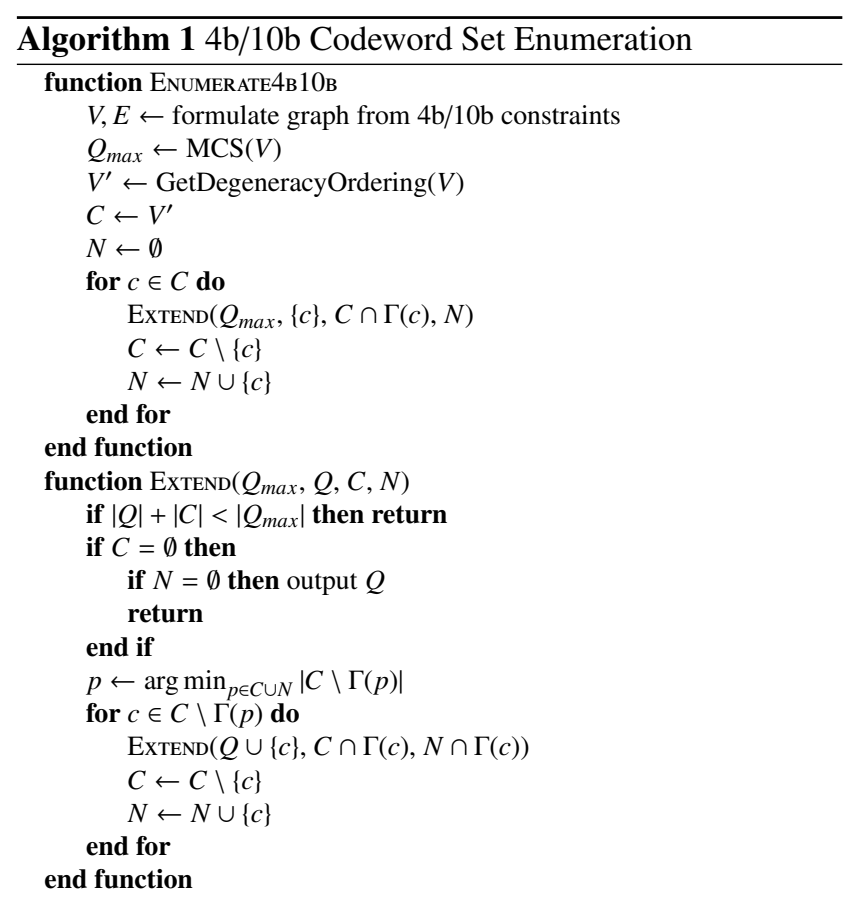

solution to the codeword search problem above, as by definition, no clique $S^{\prime}$ such that $\left|S^{\prime}\right|>|S|$ can exist.

One question that may arise is whether there are more efficient algorithms for solving this problem. Unfortunately, there is strong evidence that this is most likely not the case. We have shown that this problem is NP-hard [1], meaning that if this problem could be solved in its general form in polynomial time, then $\mathrm{P}=\mathrm{NP}$. Indeed, the algorithm used to find the original set of codewords specified in the standard is a greedy heuristic which, while being polynomial in complexity, has no guarantees of finding the maximum set and found a set of only 18 codewords [18].

\section{Codeword Enumeration as a Maximum Clique Enu- meration Problem}

Given a set of codewords found via some MCP algorithm, a question that arises is whether such set is the only set of its size and if not, whether there are others which are more suitable for use as a line code. Multiple maximum cliques may exist; the problem of enumerating all of them is referred to as the Maximum Clique Enumeration Problem (MCE). In order to solve MCE, we use the Intelligent Backtracking algorithm, proposed by Eblen et al. [2] with the MCS algorithm, proposed by Tomita et al. [19], instead of using MaximumCliqueFinder as the maximum clique algorithm. Additionally, we apply a degeneracy ordering of the vertices as a preprocessing step as proposed by Eppstein and Strash [20]. We show the full algorithm in Algorithm 1. The algorithm was implemented in the Rust programming language, executed on a machine specified in Table 1, and took 7.11 seconds.

We present the sets of codewords found in Tables 2, 


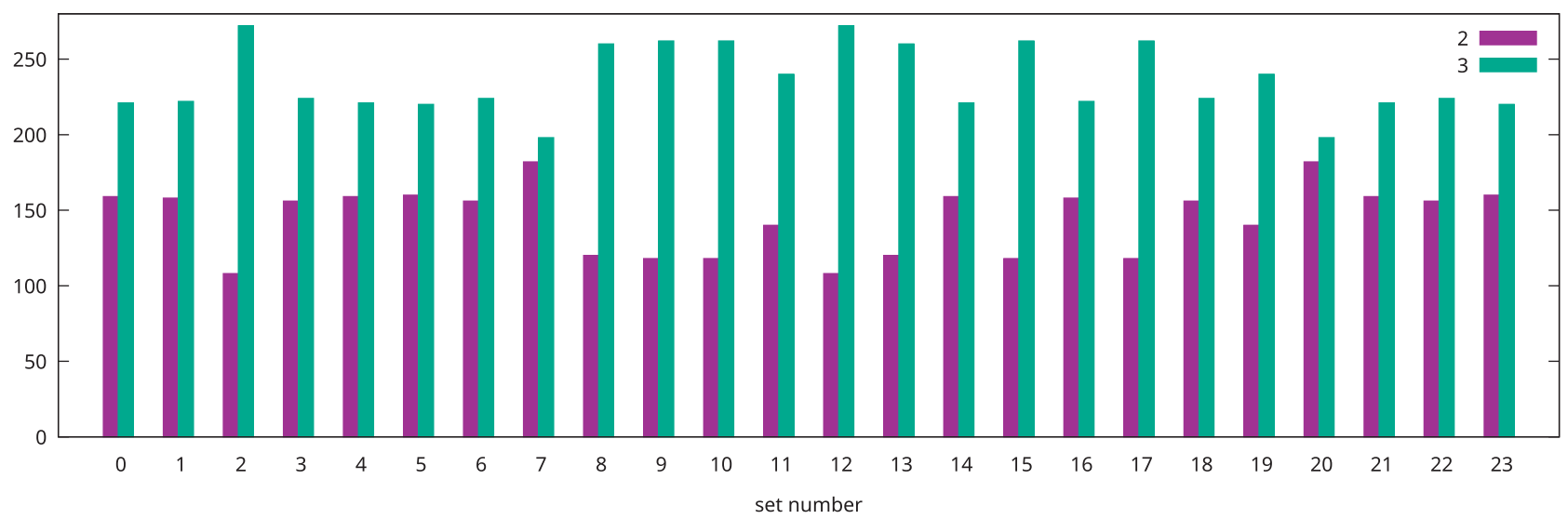

Fig. 2 The distribution of the run length of concatenated codewords. The $4 b / 10 b$ line code specifies that this value must be equal or less than five for all pairs of codewords in order to enable clock recovery.

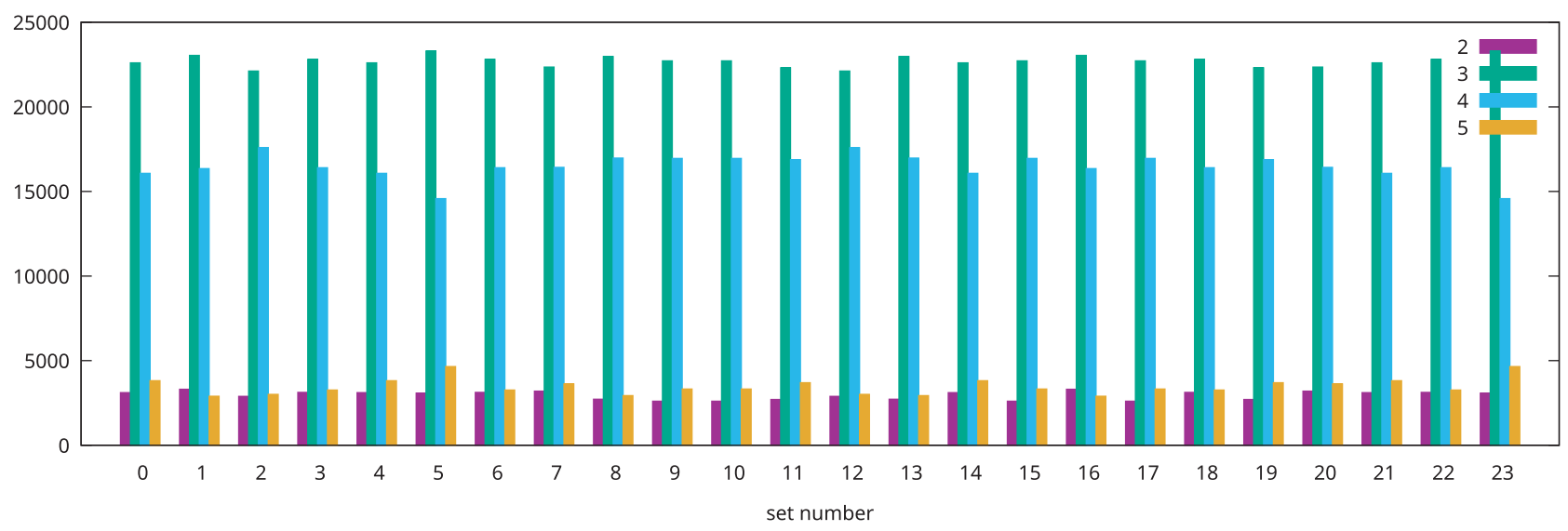

Fig. 3 The distribution of the run length of concatenated codewords, when two bits at least four bits apart, have been flipped. The $4 \mathrm{~b} / 10 \mathrm{~b}$ line code specifies that this value must be equal or less than five for all pairs of codewords in order to preserve clock recovery in the presence of noise. Ideally, this should hold for all double bit flips, but strengthening this constraint further will reduce the size of candidate codewords to the point where it becomes impossible to map four bits to them.

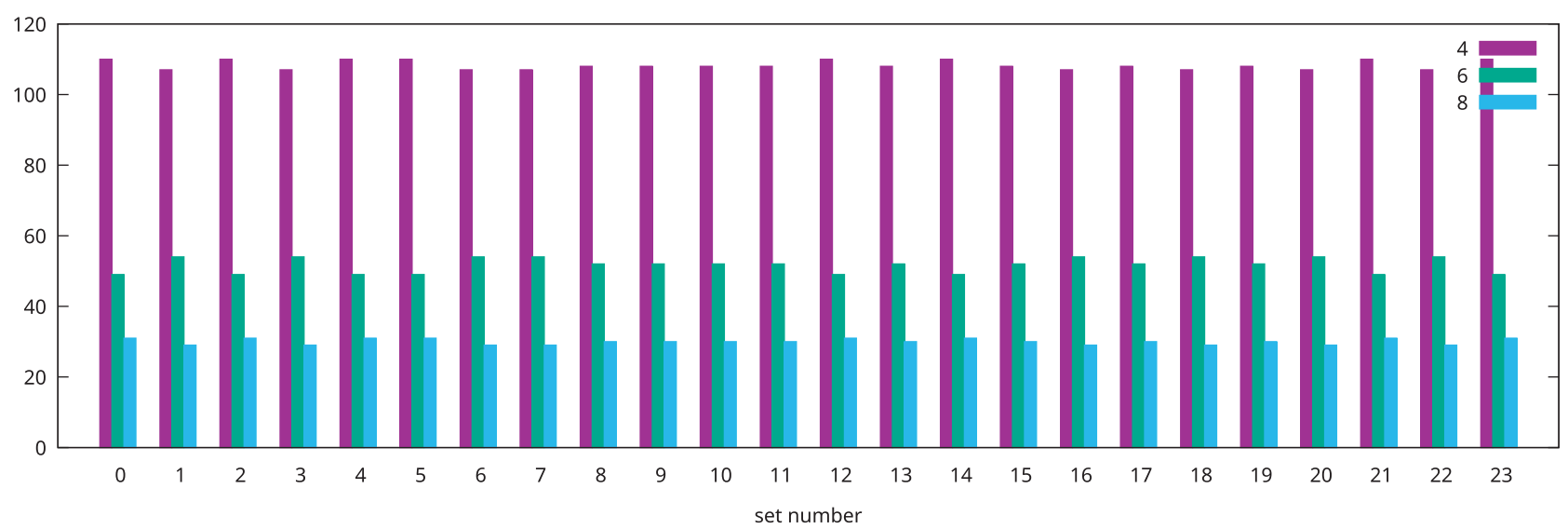

Fig. 4 The distribution of the hamming distance between codewords. The $4 \mathrm{~b} / 10 \mathrm{~b}$ line code specifies this value must be greater or equal than four in order to implement error correction. As codewords have the same number of zeros and ones, hamming distances only take even values.

3, and 4. As there was significant overlap among the sets, we number and list the union of all codewords appearing in maximum cliques found in Tables 2 and 3, and refer to codewords by number when listing them in Table 4 . 
Table 5 The canonical $4 \mathrm{~b} / 10 \mathrm{~b}$ translation table based on codeword set 7 .

\begin{tabular}{|c|c|}
\hline input & output \\
\hline 0000 & 0010110110 \\
\hline 0001 & 0011001101 \\
\hline 0010 & 0011010011 \\
\hline 0011 & 0011101010 \\
\hline 0100 & 0100110011 \\
\hline 0101 & 0101010101 \\
\hline 0110 & 0101011010 \\
\hline 0111 & 0101100110 \\
\hline 1000 & 0101101001 \\
\hline 1001 & 0110001110 \\
\hline 1010 & 0110011001 \\
\hline 1011 & 0110100101 \\
\hline 1100 & 1000101110 \\
\hline 1101 & 1000111001 \\
\hline 1110 & 1001001011 \\
\hline 1111 & 1001010110 \\
\hline setup & 1001100101 \\
\hline idle & 1010010101 \\
\hline ack & 1010011010 \\
\hline command & 1010100011 \\
\hline
\end{tabular}

Eblen et al. [2] empirically observes that there exists a nonempty set of "essential" vertices shared by all maximum cliques for most graphs and proposes a pruning method based on this fact for use prior to running MCE algorithms. Notably, this is not the case for our graph, despite significant overlap among maximum cliques found, with vertices 6 and 91 both being shared among 12 cliques and coming the closest to being "essential". Furthermore, these two vertices form the smallest instance of a maximum clique cover $V^{\prime} \subseteq V$ where all maximum cliques in $G$ contain a vertex in $V^{\prime}$. This can be explained by the constraints being agnostic to order and inversion; that is, given a set of codewords conformant to these constraints, the set of codewords obtained by reversing or inverting all codewords will also result in a conformant codeword set. Thus, if a codeword set is not equal to itself under either reversion or inversion, the essential set will be empty. This can be confirmed by observing that the codeword corresponding to vertex 6 is both the inverted and reversed version of the codeword corresponding to vertex 91 .

\section{Evaluation and Selection}

We evaluate the sets found based on the $4 \mathrm{~b} / 10 \mathrm{~b}$ line code's constraints with respect to each other mentioned in Sect. 4 The distribution of these values for all candidate sets are shown in Figs. 2, 3, and 4. As Fig. 2 shows, set 7 and 20 have the shortest run lengths on average, with 182 instances out of a total 380 having a run length of 2 . These two sets are similarly favorable across all remaining measures, with negligible differences between cliques as seen in Figs. 3 and 4. Thus, we present set 7, somewhat arbitrarily, as the canonical 4b/10b line code in Table 5. Notably, the codeword set found in [1] corresponds to set 8 , which has a worse run length distribution when compared to set 7 as shown in Fig. 2.

\section{8. \#P-hardness of Maximum Clique Enumeration}

As MCP is NP-hard, MCE is clearly also NP-hard, but it remains to be seen whether it can be classified as an instance of a more specific complexity class. Here, we show the following:

\section{Theorem 1. Maximum Clique Enumeration is \#P-hard.}

Proof. We show this by reduction from \#3SAT. Given an instance of 3SAT, under Karp's formulation as a graph [21] for the proof of the NP-completeness of the clique decision problem, enumerating all maximum cliques will enumerate all assignments that satisfy the formula. Thus, counting the number of enumerated maximum cliques will yield the answer to \#3SAT. As this reduction can be performed in polynomial time, MCE is \#P-hard.

\section{Conclusion}

We have presented all maximum sets of codewords possible for the $4 b / 10 b$ line code found via formulation of codeword search and enumeration as instances of maximum clique problems, and chosen a canonical set based on qualitative evaluations. The $4 \mathrm{~b} / 10 \mathrm{~b}$ line code has substantial application in real-time communication due to its ease of implementation due to its simplicity and codeword-level compatibility with $8 \mathrm{~b} / 10 \mathrm{~b}$ enabling switching between line codes depending on the use case. Additionally, we have shown that maximum clique enumeration belongs to the \#P-hard complexity class. We believe that this approach of finding and enumerating codewords under various constraints has wide applications and deserves further study.

\section{References}

[1] M. Takeda and N. Yamasaki, "Finding the maximum number of symbols for the $4 \mathrm{~b} / 10 \mathrm{~b}$ line code with error correction," 2019 Seventh International Symposium on Computing and Networking Workshops, 2019.

[2] J.D. Eblen, C.A. Phillips, G.L. Rogers, and M.A. Langston, "The maximum clique enumeration problem: Algorithms, applications, and implementations," BMC Bioinform., vol.13, p.S5, BioMed Central, 2012.

[3] G.C. Buttazzo, Hard Real-Time Computing Systems: Predictable Scheduling Algorithms and Applications, Springer Science \& Business Media, 2011.

[4] K. Tindell, A. Burns, and A.J. Wellings, "Analysis of hard real-time communications,” Real-Time Syst., vol.9, no.2, pp.147-171, 1995.

[5] "802.3-2018 - IEEE Standard for Ethernet," 2018.

[6] "Universal Serial Bus 3.1 Specification," 2013.

[7] I.S. Reed and G. Solomon, "Polynomial codes over certain finite fields,” J. Soc. Ind. Appl. Math., vol.8, no.2, pp.300-304, 1960.

[8] K. Suito, R. Ueda, K. Fujii, T. Kogo, H. Matsutani, and N. Yamasaki, "The dependable responsive multithreaded processor for distributed real-time systems," IEEE Micro, vol.32, no.6, pp.52-61, 2012.

[9] K. Suito, T. Kogo, H. Matsutani, and N. Yamasaki, "Design and implementation of dependable communication mechanism on responsive link for distributed real-time systems," IPSJ Journal of Information Processing, vol.53, no.12, pp.2728-2739, 2012 (in Japanese). 
[10] "Dependable Line Code with Error Correction Capability: 4b/10b," IPSJ-TS 0006:2003.

[11] "Multimedia systems and equipment - Multimedia signal transmission - Dependable line code with error correction," IEC TR 63094:2017.

[12] B. Raahemi, "Error correction on 64/66 bit encoded Links," Canadian Conference on Electrical and Computer Engineering, 2005, pp.412-416, IEEE, 2005.

[13] D.T. Carney and E.W. Chandler, "Error correction coding for a serial digital multi-gigabit communication system," 2004 IEEE Electro/Information Technology Conference, pp.33-41, Aug. 2004.

[14] G. Papotti, "Architectural studies of a radiation-hard transceiver asic in 0.13 um cmos for digital optical links in high energy physics applications," Technical Report, CERN, 2007.

[15] Q. Wu and J.K. Hao, "A review on algorithms for maximum clique problems,” Eur. J. Oper. Res., vol.242, no.3, pp.693-709, 2015.

[16] T. Etzion and P.R. Ostergard, "Greedy and heuristic algorithms for codes and colorings," IEEE Trans. Inf. Theory, vol.44, no.1, pp.382388, 1998.

[17] I.M. Bomze, M. Budinich, P.M. Pardalos, and M. Pelillo, "The maximum clique problem," Handbook of Combinatorial Optimization, pp.1-74, Springer, 1999.

[18] N. Yamasaki, "Conversion method of transmission line code for digital communication, and code generation method for the conversion method," Japanese Patent \#P5900850, 2016.

[19] E. Tomita, Y. Sutani, T. Higashi, S. Takahashi, and M. Wakatsuki, "A simple and faster branch-and-bound algorithm for finding a maximum clique," International Workshop on Algorithms and Computation, pp.191-203, Springer, 2010.

[20] D. Eppstein and D. Strash, "Listing all maximal cliques in large sparse real-world graphs," International Symposium on Experimental Algorithms, pp.364-375, Springer, 2011.

[21] R.M. Karp, "Reducibility among combinatorial problems," Complexity of Computer Computations, pp.85-103, Springer, 1972.

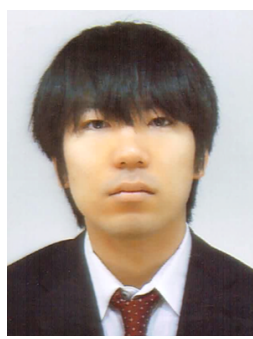

Masayuki Takeda is an undergraduate student at the Department of Information and Computer Science at Keio University, expected to receive his B.S. degree in 2020. His research interest include real-time systems and clique problems.

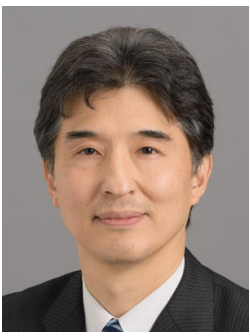

Nobuyuki Yamasaki is a Professor in the Department of Information and Computer Science at Keio University. He received his B.S., M.S., and Ph.D. degrees from Keio University in 1991, 1993, and 1996, respectively. His research interests include processor architecture, parallel and distributed systems, real-time systems, real-time communication, System-onChip (SoC), and robotics. 